\title{
Aspectos ecológicos de la comunidad fitoperifítica en el río Anchique, cuenca andina colombiana
}

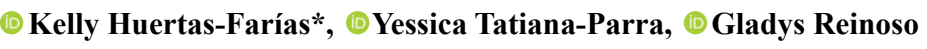 \\ Grupo de Investigación en Zoología - GIZ, Universidad del Tolima.
}

\begin{abstract}
Resumen
El fitoperifiton contribuye significativamente a la dinámica de los ecosistemas, ya que oxigena el agua, aporta materia orgánica y proporciona abrigo y alimento a los organismos acuáticos. En ese contexto, el presente estudio se orientó a determinar su dinámica en el río Anchique, cuenca andina colombiana de gran relevancia. Los muestreos se hicieron en cuatro puntos localizados a lo largo del río en temporadas meteorológicas contrastantes mediante la metodología de raspados de superficie en dos sustratos naturales (roca y tronco). Se registró una densidad relativa de 5.070 organismos $/ \mathrm{cm}^{2}$ distribuidos en cinco divisiones, nueve clases y 59 géneros, siendo la división Bacillariophyta la más abundante. La estación E1 presentó los valores más altos de diversidad efectiva (q:0, q:1 y q:2), mientras que la E4 registró los valores más bajos. El sustrato de roca presentó las mayores densidades relativas. Se encontró una relación inversa entre el caudal y la densidad relativa de los organismos, con altas densidades relativas en los meses de pocas precipitaciones y menores en los meses de intensas precipitaciones. Se concluyó que las temporadas meteorológicas y el grado de intervención antrópica son algunos de los factores relevantes en la composición y estructura de la comunidad fitoperifítica en la cuenca del río Anchique. (C) 2019. Acad. Colomb. Cienc. Ex. Fis. Nat.
\end{abstract}

Palabras clave: Fitoperifiton; Río Anchique; Ecología; Composición; Parámetros fisicoquímicos.

Ecological aspects of the phytoperiphytic community in the Anchique River, Colombian Andean basin

\begin{abstract}
Phytoperifiton contributes significantly to the dynamics of the ecosystem since it oxygenates the water and provides organic matter, shelter, and nourishment to aquatic organisms. In this context, we conducted the present study to determine its dynamics in the Anchique River, an important Colombian Andean basin. The samplings were carried out in four points located along the river in contrasting weather seasons using the methodology of surface scraping on two natural substrates (rock and trunk). We registered a relative density of 5,070 organisms $/ \mathrm{cm} 2$ distributed in five divisions, nine classes and 59 genera of which the Bacillariophyta division was the most abundant. Station E1 presented the highest values of richness, diversity, and dominance, while E4 recorded the lowest values. The rock substrate presented the highest relative densities. We found an inverse relationship between the flow and the relative density of the organisms with high relative densities during the months of low rainfall and the lowest values during the months of high rainfall. We concluded that the meteorological seasons and the degree of anthropic intervention are some of the relevant factors in the composition and structure of the phytoperiphytic community in the Anchique River basin. (C) 2019. Acad. Colomb. Cienc. Ex. Fis. Nat.
\end{abstract}

Key words: Phytoperiphyton; Anchique River; Structure; Composition; Physicochemical parameters.

\section{Introducción}

Los ríos son ecosistemas dulceacuícolas de gran relevancia, ya que albergan una abundante y diversa biota en la que se destaca la comunidad de algas perifíticas, las cuales son productoras primarias dominantes en los cursos de agua (Gómez, et al., 2009), presentan altas tasas de renovación y poseen estrategias oportunistas que les permiten establecerse con éxito en diversos hábitats (Goring \& Biggs, 1996). Su importancia se atribuye a su capacidad para capturar la energía lumínica, a su papel como fuente de alimento de varios organismos acuáticos y a su transformación en compuestos orgánicos, característica que la posiciona en un nivel trófico importante (Roldán, 1992; Biggs \& Smith, 2002).

Las algas perifíticas, principalmente las diatomeas, se caracterizan por su sensibilidad específica a una variedad de condiciones ecológicas, físicas y químicas en los arroyos y

\footnotetext{
*Correspondencia:

Kelly Huertas-Farías, huefar@hotmail.com

Recibido: 21 de agosto de 2018

Aceptado: 13 de marzo de 2019

Editor: Sandra Baena Garzón
} 
ríos (Lobo, et al., 2016), por lo tanto, pueden usarse para el monitoreo de la contaminación ambiental mediante la determinación de sus diferentes grados de tolerancia a partir de los valores tróficos (Lobo, et al., 2015), ya que responden de manera rápida a diferentes tensores ambientales $\mathrm{y}$ se establecen de forma fija en un sustrato (Masseret, et al., 1998; Wu, 1999; Smith \& Smith, 2001; Harding, et al., 2005; Arcos \& Gómez, 2006).

Debido a las dinámicas antropogénicas, el deterioro de los recursos hídricos es progresivo y pone en riesgo la biota que albergan. La expansión de la frontera agrícola y ganadera, el establecimiento y puesta en marcha de industrias de gran producción y la creciente densidad de la población humana, entre otros, han sido los factores más determinantes en la transformación del paisaje, pues afectan negativamente la estructura, la función y la composición de los ecosistemas lóticos mediante la alteración de sus características físicas y ambientales con la consecuente pérdida de su biodiversidad (Rivera, et al., 2010; Smucker \& Vis, 2011).

De ahí la necesidad de realizar estudios sobre los ecosistemas acuáticos, su dinámica y la relación entre su diversidad y la capacidad de adaptación de las diferentes especies a los cambios estructurales o fisicoquímicos de origen natural o antropogénico, cuyo conocimiento es una herramienta relevante para comprender los mecanismos de respuesta de las comunidades ante posibles alteraciones del ambiente (Patrick, 1988; Rivera \& Donato, 2008).
Dada la importancia del río Anchique para las poblaciones del municipio de Natagaima en el departamento del Tolima, pues constituye su principal fuente hídrica, y del papel del fitoperifiton en la dinámica de los ecosistemas dulceacuícolas, se propuso la presente investigación para evaluar la estructura y la composición de las microalgas perifíticas en esta importante cuenca andina. Los resultados obtenidos son un aporte importante para futuros estudios taxonómicos y de bioindicación y constituyen una información necesaria para el diseño de planes y programas de manejo y conservación de la cuenca, incluida su biota fitoperifítica. Además, establecen la línea de base de la taxonomía de las algas perifíticas en Colombia y contribuyen a determinar el estado ecológico de las cuencas andinas colombianas dando cuenta de su abundancia, densidad y diversidad.

\section{Materiales y métodos}

Área de estudio. Se seleccionaron cuatro estaciones de muestreo ubicadas a lo largo de la cuenca del río Anchique (E1: 03'33'11,7"'; E2: 03³3'40,0"; E3: 03³5'10,1"; E4: 0334'28,7" N, y E1: 7509'49,7'; E2: 7508'31,2”;

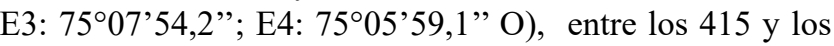
333 m s.n.m. y con una distancia lineal entre ellas de $3 \mathrm{~km}$ aproximadamente (Figura 1). La estación E1 se ubica en la parte alta de la cuenca, caracterizada por ser un cuerpo de agua con poco caudal y alta incidencia de material alóctono; las estaciones E2 y E3 son balnearios y puntos empleados

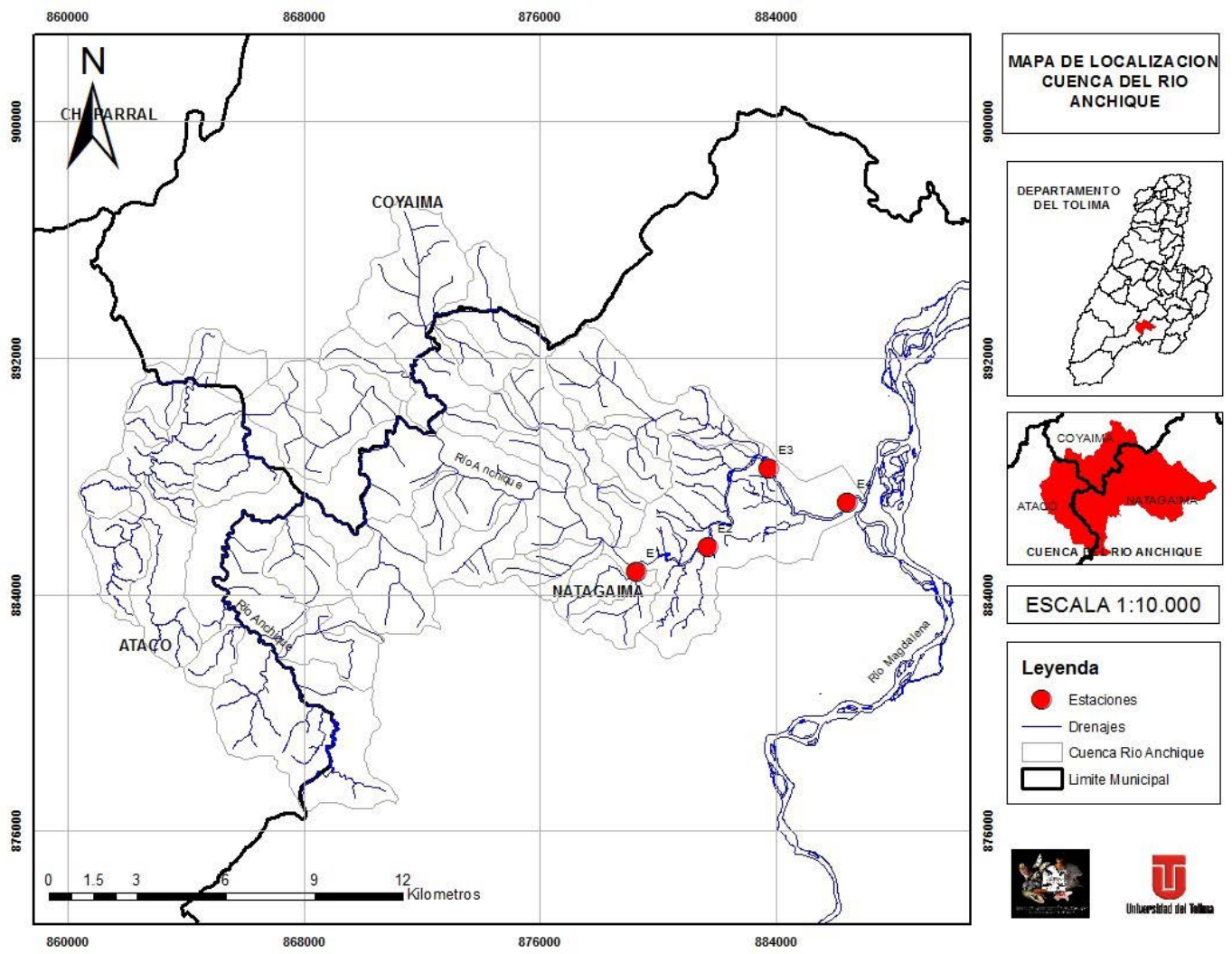

Figura 1. Estaciones de muestreo en el río Anchique, cuenca andina colombiana 
para lavar, en tanto que la estación E4 está en un punto de menor altitud muy intervenido, pues es un paso vehicular y de ganado. Se hicieron cinco muestreos durante un ciclo hidrológico: desde mayo del 2015 hasta julio del 2016, y se tomó la información de los registros históricos y de los últimos 20 años de las lluvias hechos por el Instituto de Hidrología, Meteorología y Estudios Ambientales (IDEAM) en la cuenca.

Toma de muestras biológicas. Para la recolección del material perifítico se seleccionaron dos tipos de sustratos naturales en inmersión (roca y tronco) en cada estación de muestreo mediante raspados de superficie en un área de 10 $\mathrm{cm}^{2}$ de cada sustrato con la ayuda de cepillos plásticos según la metodología recomendada por Tümpling \& Friedrich (1999). Las muestras se fijaron con una solución preservante de formol al $10 \%$ y lugol siguiendo la metodología recomendada por Weber (1968).

Los individuos se contaron usando la fórmula propuesta por Hauer \& Lambert (2007) en la cual la densidad equivale al número de individuos por área de superficie $\left(\mathrm{cm}^{2}\right)$. Para la identificación taxonómica de las algas se siguieron las claves de Needham \& Needham (1978), Kramer \& Lange-Bertalot (1979), Streble \& Krauter (1987), Lopretto \& Tell (1995), Ramírez (2000), Wehr \& Sheat (2003) y Bellinger \& Sigee (2011), así como las indicaciones de las publicaciones de Martínez \& Donato (2003), Díaz-Quirós \& Rivera-Rondón (2004), y Bicudo \& Meneses (2006). Además, como respaldo de la determinación taxonómica de las algas se emplearon las bases de datos electrónicas Algaebase y Diatombase (Guiry \& Guiry, 2013; Kociolek, et al., 2016).

Análisis bacteriológicos y fisicoquímicos. Se hicieron mediciones in situ de la temperatura del agua y del ambiente, de la profundidad y del ancho y la velocidad de la corriente; las descripciones geomorfológicas del cuerpo de agua y las características de la zona ribereña se registraron en fichas de campo. Posteriormente, se recolectaron muestras de agua superficial y en contra de la corriente en frascos plásticos con capacidad para $2.000 \mathrm{ml}$. Se analizaron el $\mathrm{pH}$, la conductividad eléctrica $(\mu \mathrm{S} / \mathrm{cm})$, el oxígeno disuelto ( $\mathrm{mg} \mathrm{O}_{2} / \mathrm{l}$ ), la turbidez (UNF), la alcalinidad total y la dureza $\left(\mathrm{mg} \mathrm{CaO}_{3} / \mathrm{l}\right)$, los nitratos $\left(\mathrm{mg} \mathrm{NO}_{3} / \mathrm{l}\right)$, los fosfatos (mg PO4/l), los sólidos totales ( $\mathrm{mg} / \mathrm{l})$, la demanda química de oxígeno (DQO: $\mathrm{mg} \mathrm{O}_{2} / \mathrm{l}$ ) y la demanda bioquímica de oxígeno $\left(\mathrm{DBO}_{5}: \mathrm{mg} \mathrm{O}_{2} / \mathrm{l}\right)$, el fosforo total $(\mathrm{P})$ y los sulfatos $\left(\mathrm{mgSO}_{4} / 1\right)($ American Public Health Association - APHA, American Water Works Association - AWWA, Water Environment Federation - WEF, 2012). Para la determinación de los parámetros bacteriológicos, se tomaron muestras de agua superficialmente y en contra corriente en frascos de vidrio con capacidad para $300 \mathrm{ml}$, las cuales se rotularon y preservaron en frío para el posterior análisis de coliformes totales (UFC/100 ml) y fecales (UFC/100ml) en el laboratorio de análisis químico LASEREX de la Universidad del Tolima (Roldán, 1992; Ramírez \& Viña, 1998; Roldán \& Ramírez, 2008) (Tabla 1).
Análisis de variables fisicoquímicas. Se utilizó la prueba de análisis de normalidad de Shapiro Wilks para evaluar la distribución de las variables fisicoquímicas a nivel espacial y temporal y, posteriormente, el análisis de varianza no paramétrico de Kruskal Wallis para determinar la existencia de diferencias significativas a nivel temporal y espacial. Las pruebas se hicieron con el programa estadístico InfoStat (Di rienzo, et al., 2013).

Análisis de correlación canónica. Se hizo el análisis de correlación canónica (ACC) para establecer la existencia y el grado de asociación entre las variables fisicoquímicas y la comunidad fitoperifítica del río Anchique con el programa Canoco, versión 4.5 (Braak \& Smilauer, 2004).

Análisis estadísticos. Se determinó la densidad relativa a partir del número de individuos recolectados de cada género y su relación con el número total de individuos de la muestra; para establecer la diversidad se aplicó la serie de los números efectivos de Hill (1973). Se hizo un análisis de Shapiro Wilks para evaluar la distribución normal en los datos. Para la comparación de las medias de estos índices ecológicos se empleó un ANOVA de una vía para determinar si existían diferencias estadísticamente significativas entre los índices evaluados. Se utilizó la prueba de diferencia mínima significativa de Fisher para comparar entre muestreos y estaciones. Se aplicó el índice de similaridad de Bray Curtis para establecer una medida de la diferencia entre las abundancias de cada género en las diferentes estaciones de muestreo (Brower \& Zar, 1984). En el análisis

Tabla 1. Métodos analíticos para el cálculo de parámetros químicos

\begin{tabular}{ll}
\hline Parámetros & Método analítico \\
\hline $\mathrm{pH}$ & Potenciométrico/pH métrico \\
\hline $\begin{array}{l}\text { Conductividad eléctrica } \\
\text { Oxígeno disuelto }\end{array}$ & Potenciométrico/conductimétrico \\
\hline $\begin{array}{l}\text { Porcentaje de saturación } \\
\text { de oxígeno }\end{array}$ & Yodométrico \\
\hline Turbidez & Espectrofotométrico/UV-Vis \\
\hline Alcalinidad total & Electrodo selectivo/neutralización \\
\hline $\begin{array}{l}\text { Dureza } \\
\text { Nitratos }\end{array}$ & Electrodo selectivo/complexiométrico \\
\hline Fosfatos & Espectrofotométrico/UV \\
\hline Sólidos totales & Espectrofotométrico/Vis \\
\hline $\begin{array}{l}\text { Demanda química de } \\
\text { oxígeno (DQO) }\end{array}$ & Gravimétrico/evaporación \\
\hline $\begin{array}{l}\text { Demanda bioquímica } \\
\text { de oxígeno (DBO5 ) }\end{array}$ & Incubación y electrometría \\
\hline Fósforo total & Espectrofotométrico/Vis \\
\hline $\begin{array}{l}\text { Sulfatos } \\
\text { Coliformes totales }\end{array}$ & Espectrofotométrico/Vis \\
\hline Coliformes fecales & Filtración por membrana \\
\hline
\end{tabular}


de escalamiento multidimensional no métrico (non-metric multidimensional scaling, NMDS) se implementó la distancia de Bray Curtis con el paquete Primer-6 (Clarke \& Gorley, 2006) para evaluar el ensamblaje de las comunidades fitoperifíticas a nivel temporal y por sustratos.

\section{Resultados}

Se obtuvo una densidad total de 5,070 organismos $/ \mathrm{cm}^{2}$ distribuidos en cinco divisiones, nueve clases, 24 órdenes, 43 familias y 59 géneros (Tabla 2). La división Bacillariophyta registró la mayor densidad relativa $(59,88 \%)$, seguida de Charophyta $(16,24 \%)$, en tanto que Euglenophyta tan solo registró un $0,07 \%$ de representatividad (Figura 2). A nivel de género, Synedra tuvo la mayor densidad relativa $(19,08 \%)$, seguido de Surirella (12,3\%), Navicula (9,52\%), Cosmarium $(9,6 \%)$, Cymbella (7,13\%) y Oscillatoria (6,92\%), en tanto que géneros como Desmidium, Diploneis y Euastrum registraron las densidades más bajas $(0,03 \%)$.

A nivel espacial la estación que presentó la mayor densidad relativa fue la E1 (nacimiento) con 36,31\%, seguida de E3 (puente) con 26,60\%, E2 (balneario) con 25,23\% y, por último, la E4 (desembocadura) con 11,84\%. En cuanto a la temporalidad es de resaltar que el mes de diciembre de 2015 fue el que presentó la mayor densidad relativa, con $27,47 \%$, seguido del mes de julio de 2015 , con $27,24 \%$, es decir, en los meses de pocas precipitaciones, en tanto que en los meses con altas precipitaciones se presentaron las menores densidades relativas: $6,83 \%$ en abril y $22,50 \%$ en julio de 2016 (Figura 3).

En general, el sustrato de roca registró la mayor densidad relativa, con un promedio de 58,48 \%, y el sustrato de tronco tuvo una de 41,51\%. Ambos sustratos obtuvieron una composición fitoperifítica cercana en cuanto al número de taxones, con 47 géneros en la roca y 46 en los troncos.

Variables fisicoquímicas. La mayoría de los datos fisicoquímicos evidenciaron normalidad (Shapiro Wilks: $\mathrm{p}>0,05$ ), con excepción de los sólidos totales y los fosfatos en los muestreos, así como la turbidez, el porcentaje de saturación de oxígeno y el $\mathrm{pH}$ en las estaciones. Por otra parte, el análisis del ANOVA y la prueba de Kruskal Wallis no evidenciaron diferencias estadísticamente significativas entre los parámetros fisicoquímicos ( $\mathrm{p}>0,9999)$.

Tabla 2. Composición taxonómica y densidad de las microalgas perifíticas en la cuenca del río Anchique

\begin{tabular}{|c|c|c|c|c|}
\hline Filo & Orden & Género & Densidad & Densidad relativa \\
\hline \multirow[t]{23}{*}{ Bacillariophyta } & Bacillariales & Nitzschia & 252,08 & 4,97 \\
\hline & \multirow[t]{2}{*}{ Coscinodiscales } & Cocconeis & 9,62 & 0,19 \\
\hline & & Actinocyclus & 5,77 & 0,11 \\
\hline & \multirow[t]{3}{*}{ Cymbellales } & Cymbella & 361,76 & 7,13 \\
\hline & & Gomphonema & 34,64 & 0,68 \\
\hline & & Rhoicosphenia & 11,55 & 0,23 \\
\hline & Fragilariales & Fragilaria & 25,02 & 0,49 \\
\hline & Licmophorales & Synedra & 967,89 & 19,09 \\
\hline & \multirow[t]{2}{*}{ Melosirales } & Hyalodiscus & 34,64 & 0,68 \\
\hline & & Melosira & 5,77 & 0,11 \\
\hline & \multirow[t]{8}{*}{ Naviculales } & Amphipleura & 5,77 & 0,11 \\
\hline & & Caloneis & 9,62 & 0,19 \\
\hline & & Diploneis & 1,92 & 0,04 \\
\hline & & Frustulia & 40,41 & 0,80 \\
\hline & & Gyrosigma & 61,58 & 1,21 \\
\hline & & Luticola & 1,92 & 0,04 \\
\hline & & Navicula & 482,98 & 9,53 \\
\hline & & Pinnularia & 44,26 & 0,87 \\
\hline & Rhopalodiales & Rhopalodia & 34,64 & 0,68 \\
\hline & Surirellales & Surirella & 631,15 & 12,45 \\
\hline & \multirow[t]{2}{*}{ Tabellariales } & Tabellaria & 1,92 & 0,04 \\
\hline & & Tetracyclus & 7,70 & 0,15 \\
\hline & Thalassiophysales & Amphora & 3,85 & 0,08 \\
\hline
\end{tabular}




\begin{tabular}{|c|c|c|c|c|}
\hline \multirow[t]{16}{*}{ Charophyta } & \multirow[t]{8}{*}{ Desmidiales } & Closterium & 55,80 & 1,10 \\
\hline & & Cosmarium & 486,83 & 9,60 \\
\hline & & Desmidium & 1,92 & 0,04 \\
\hline & & Euastrum & 1,92 & 0,04 \\
\hline & & Gonatozygon & 150,09 & 2,96 \\
\hline & & Micrasteria & 13,47 & 0,27 \\
\hline & & Penium & 5,77 & 0,11 \\
\hline & & Staurastrum & 1,92 & 0,04 \\
\hline & \multirow[t]{8}{*}{ Zygnematales } & Ancylonema & 19,24 & 0,38 \\
\hline & & Cylindrocystis & 5,77 & 0,11 \\
\hline & & Mesotaenium & 1,92 & 0,04 \\
\hline & & Mougeotia & 1,92 & 0,04 \\
\hline & & Netrium & 34,64 & 0,68 \\
\hline & & Roya & 9,62 & 0,19 \\
\hline & & Spirogyra & 23,09 & 0,46 \\
\hline & & Spirotaenia & 9,62 & 0,19 \\
\hline \multirow[t]{6}{*}{ Chlorophyta } & Chlorellales & Oocystis & 26,94 & 0,53 \\
\hline & \multirow[t]{3}{*}{ Sphaeropleales } & Ankistrodesmus & 3,85 & 0,08 \\
\hline & & Hydrodictyon & 7,70 & 0,15 \\
\hline & & Scenedesmus & 48,11 & 0,95 \\
\hline & Trebouxiales & Botryococcus & 300,18 & 5,92 \\
\hline & Ulotrichales & Ulothrix & 1,92 & 0,04 \\
\hline \multirow[t]{13}{*}{ Cyanobacteria } & Synechococcales & Merismopedia & 26,94 & 0,53 \\
\hline & \multirow[t]{4}{*}{ Chroococcales } & Aphanothece & 1,92 & 0,04 \\
\hline & & Chroococcus & 5,77 & 0,11 \\
\hline & & Gloeocapsa & 1,92 & 0,04 \\
\hline & & Microcystis & 305,95 & 6,03 \\
\hline & \multirow[t]{3}{*}{ Nostocales } & Anabaena & 63,50 & 1,25 \\
\hline & & Anabaenopsis & 7,70 & 0,15 \\
\hline & & Calothrix & 3,85 & 0,08 \\
\hline & \multirow[t]{4}{*}{ Oscillatoriales } & Gleotrichia & 1,92 & 0,04 \\
\hline & & Lyngbya & 1,92 & 0,04 \\
\hline & & Oscillatoria & 386,77 & 7,63 \\
\hline & & Phormidium & 7,70 & 0,15 \\
\hline & spirulinales & Spirulina & 1,92 & 0,04 \\
\hline Euglenophyta & Euglenales & Trachelomona & 3,85 & 0,08 \\
\hline 5 & 23 & 59 & 5070,38 & 100,00 \\
\hline
\end{tabular}

Variables químicas. Entre las variables asociadas con concentraciones de partículas disueltas en esta cuenca (turbidez, sólidos totales y oxígeno disuelto), la turbidez presentó los mayores valores en las estaciones E2, E3 (106 y 103 UNT), en tanto que en la estación E1 se presentó el menor valor (2,55 UNT). El oxígeno disuelto fue mayor en las estaciones $\mathrm{E} 1(53,45 \mathrm{mg} / \mathrm{L}), \mathrm{E} 2(59,42 \mathrm{mg} / \mathrm{L})$ y E3 $(59,46 \mathrm{mg} / \mathrm{L})$, y en la estación E4 se presentó un menor valor $(49,0 \mathrm{mg} / \mathrm{L})$.
Las variables relacionadas con el proceso de óxidoreducción registraron los mayores valores de DQO y nitratos en las estaciones E3 y E4, en la estación E2 se registraron altos valores de fosfatos, $\mathrm{y}$ en la estación $\mathrm{E} 1$ se presentó el mayor valor de $\mathrm{DBO}_{5}$.

Con respecto al proceso de mineralización, en la estación E1 se registraron los mayores valores de $\mathrm{pH}$ $(8,18)$, conductividad eléctrica $(416 \mu \mathrm{S} / \mathrm{cm})$, alcalinidad 
(171 mg de $\mathrm{CaCo} / \mathrm{l})$ y dureza (367 mg de $\mathrm{CaCo} 3 / 1)$, en tanto que en las estaciones E2 y E3 se presentaron los menores valores.

Variables físicas. En las estaciones E2 y E3 se presentó la mayor velocidad de la corriente, con 0,70 y $0,64 \mathrm{~m} / \mathrm{s}$, respectivamente, así como los valores más altos de caudal (E2: $2,37 \mathrm{~m}^{3} / \mathrm{s}$ y E3: $1,31 \mathrm{~m}^{3} / \mathrm{s}$ ), ancho (E2: $19 \mathrm{~m}$ y E3: 9,86 $\mathrm{m})$ y profundidad (E2: 0,18 $\mathrm{m}$ y E3: 0,21 $\mathrm{m}$ ), en tanto que en las estaciones E1 y E4 se registraron los valores más bajos en esas variables.

Parámetros bacteriológicos. Los valores obtenidos para coliformes fecales en las épocas de muestreo en el río Anchique oscilaron entre $98 \mathrm{UFC} / 100 \mathrm{ml}$ y $12.100 \mathrm{UFC} / 100 \mathrm{ml}$. En promedio, el mayor valor lo registro el muestreo cinco (julio de 2016) (2774,5 UFC/100 ml), el cual coincidió con la temporada de bajas precipitaciones. El muestreo dos (julio de 2015) reflejó pocos coliformes fecales (1.000 UFC/100 $\mathrm{ml}$ ), lo que coincidió con días de lluvia, mas no con una temporada completa. Según el Decreto 1594 de 1984, los valores promedios de coliformes totales y fecales en los muestreos realizados en la cuenca del río Anchique estaban dentro de los valores aceptables para el uso del agua con fines recreativos.

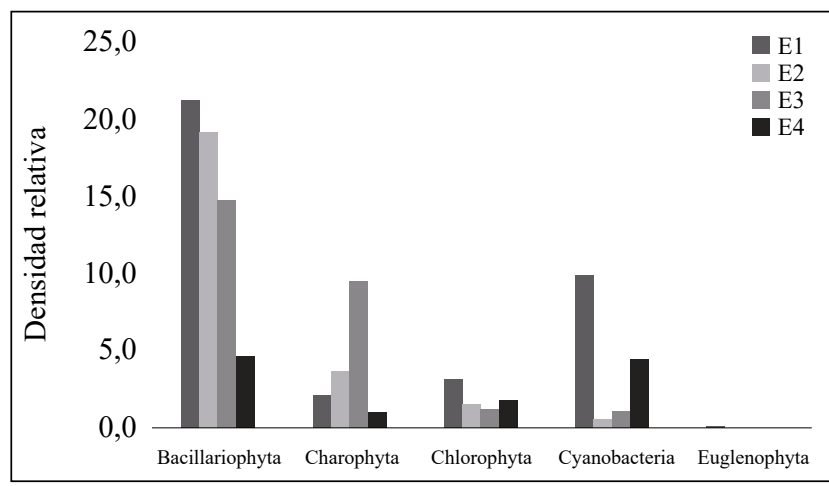

Figura 2. Densidad relativa de las divisiones del fitoperifiton presentes en las diferentes estaciones de muestreo

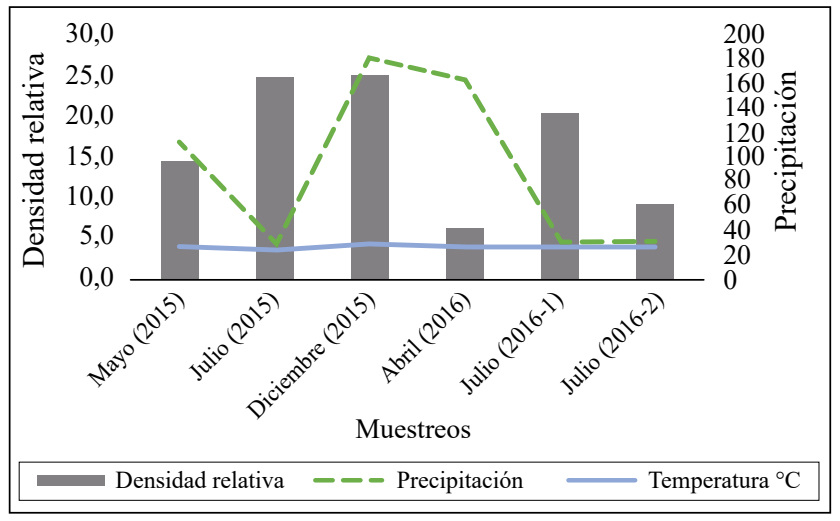

Figura 3. Climadiagrama de las condiciones hidroclimáticas en la cuenca del río Anchique
En cuanto a la variable de los coliformes totales, los valores oscilaron entre $1.400 \mathrm{UFC} / 100 \mathrm{ml}$ y 6.700 .000 UFC/100 ml. En el muestreo cinco (julio de 2016), se registró el mayor valor en promedio (1.950.000 UFC/100 $\mathrm{ml})$. El menor valor para esta variable se registró en el muestreo dos (julio de 2015), con $4.250 \mathrm{UFC} / 100 \mathrm{ml}$.

Números efectivos. Los números de diversidad de Hill, o números efectivos, indicaron que, a nivel espacial y tem-poral, en la estación E1 se registró el mayor número de géneros (medida de riqueza, q: 0), con 44, en tanto que en la estación E4 se presentó el menor número con 27 . A nivel temporal, en el muestreo dos (julio de 2015) se registró la mayor riqueza, con 44 géneros, y en el muestreo cuatro (abril de 2016), la menor, con 22 géneros. En cuanto a la medida de diversidad (q:1), que incluye todos los géneros y sus abundancias relativas, en la estación E1 se presentó una diversidad igual a la que tendría una comunidad teórica de 12 géneros. Por su parte, en las estaciones E2, E3 y E4 se registró una diversidad igual a la que tendría una comunidad de 11,10 y 11 géneros efectivos, respectivamente. Al expresar estas equivalencias, fue evidente que en la estación E1 se registró 1,09 más diversidad de géneros que en la estación E2, 1,1 más que en las estaciones E2 y E4 y 1,2 más que en la E3. En la estación E3 se presentó el 83,3 \% de la diversidad qregistrada en la E1.

A nivel temporal, la mayor diversidad de órdenes (q: 1) se registró en el muestreo dos (julio de 2015), con 14 géneros efectivos, y en el muestreo cinco se reportó la menor diversidad, con ocho géneros, es decir, tan sólo el 57,14 $\%$ de la diversidad presente en el muestreo dos. En cuanto a la medida de diversidad de los órdenes (q: 2), la mayor diversidad se registró en la estación E1, con siete géneros, en tanto que en las estaciones E2 y E3 se registró la menor diversidad, con seis géneros efectivos, y compartió el 85,71 $\%$ de la diversidad registrada en la E1. A nivel temporal, el muestreo dos (julio de 2015) presento la mayor diversidad de órdenes (q: 2), con nueve géneros efectivos, mientras que el muestreo cinco presentó la menor diversidad, con tan solo cinco géneros (Figura 4).

Análisis estadístico. Debido a que los datos biológicos no presentaron normalidad (Shapiro Wilks, $\mathrm{p}<0,05$ ), se hizo un análisis no paramétrico a nivel espacial y temporal en el cual se evidenció que las estaciones presentaron diferencias estadísticamente significativas entre sí (Kruskal Wallis: $\mathrm{p}<0,05)$. A nivel temporal no se registraron diferencias estadísticamente significativas (Kruskal Wallis: $p<0,05$ ). En este sentido, la prueba de comparación de diferencia mínima significativa de Fisher evidenció que los muestreos presentaron diferencias estadísticas significativas entre sí $(p<0,05)$.

Los valores del índice de similitud de Bray Curtis revelaron que las estaciones E2 y E3 estaban más relacionadas, pues compartían el $64 \%$ de los taxones, y que la estación E1 estaba relacionada con las estaciones E2 y E3 al compartir un $43 \%$ con la E2 y un $37 \%$ con la E3. La estación E4 presentó un menor grado de similitud con las demás 


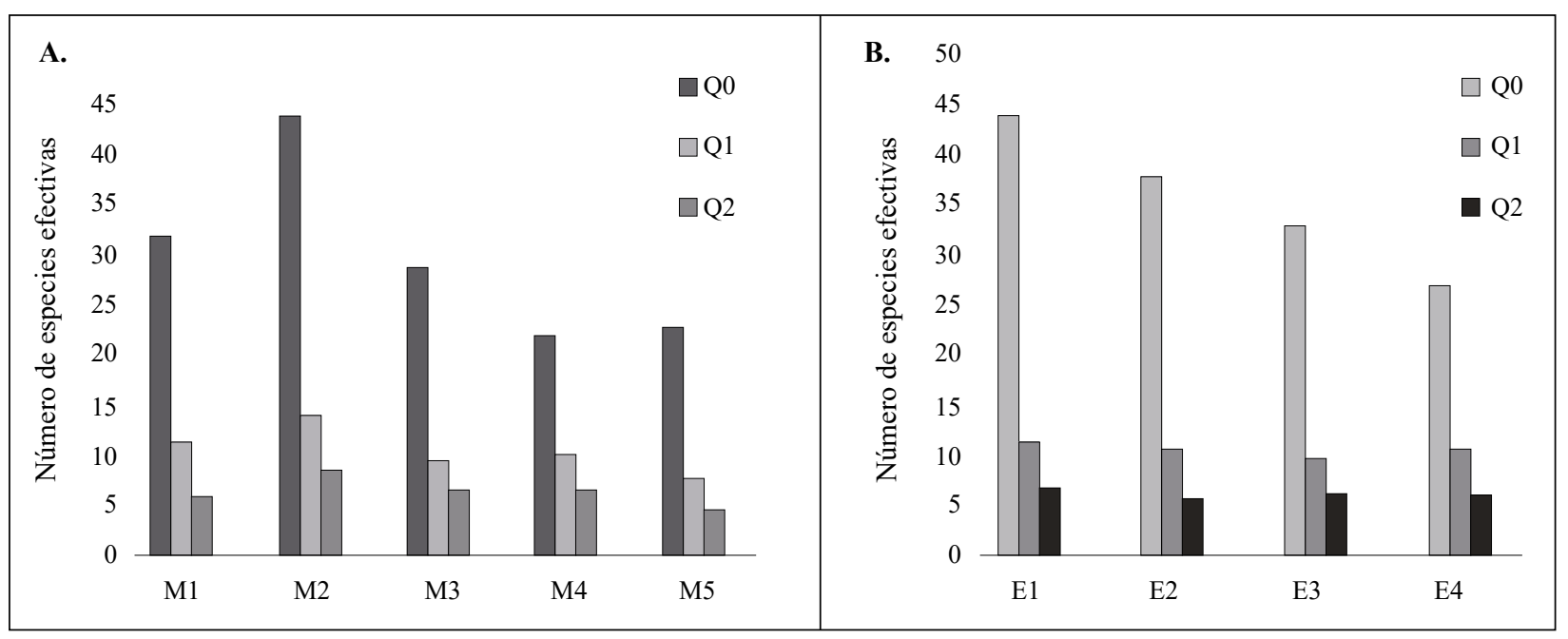

Figura 4. Índices de diversidad de Hill en las diferentes estaciones de muestreo y meses evaluados en la cuenca del río Anchique. (A) Estaciones; (B) Meses

estaciones, pues compartía el $26 \%$, el $32 \%$ y el $31 \%$ de su comunidad con las estaciones E1, E2 y E3, respectivamente. Según el análisis de escalamiento multidimensional no métrico, no hubo un ensamblaje claro en la composición de las comunidades con las variables de tipo de sustrato, estación y muestreo. Con el análisis de similitud (analysis of similarities, ANOSIM) no se encontraron diferencias significativas en las variables de tipo de sustrato, muestreos $\mathrm{y}$ estaciones $(\mathrm{p}>0,05)$.

En cuanto a la relación de las variables biológicas, se evidenció que a nivel espacial el porcentaje de saturación de oxígeno, el pH y la temperatura del agua tuvieron, al parecer, algún grado de relación con los datos biológicos (prueba de Monte Carlo: $\mathrm{p}<0,05)$. A nivel espacial, la estación E1 estaba muy relacionada con la variable del $\mathrm{pH}$, mientras que la estaciones E2 y E3 parecían relacionarse con el porcentaje de oxígeno disuelto, pues los géneros Staurastrum, Luticola, Ankistrodesmus, Spirulina, Cosmarium y Desmidium presentaron relaciones cercanas $(\mathrm{p}<0,05)$. Al parecer en la estación E4 se estableción una mayor relación con los nitratos, los coliformes totales y los fecales (Figura 5), en los géneros Mycrocystis y Gyrosigma $(\mathrm{p}<0,05)$.

A nivel temporal, el muestreo tres (diciembre de 2015) se relacionó con los géneros Luticola, Penium, Fragilaria, Merysmopedia, Mesotaenium y Cosmarium y con la variable de turbidez (Monte Carlo: $\mathrm{p}<0,05$ ); los muestreos uno (mayo de 2015) y cinco (julio de 2016-1) tuvieron una mayor relación entre sí y compartieron géneros como Staurastrum, Euastrum, Trachelomonas, Spirotaenia y Anabaena y con las variables de coliformes totales, de coliformes fecales $\mathrm{y}$ de nitratos $(\mathrm{p}<0,05)$. Algunos muestreos como el dos (julio de 2015) no evidenciaron relaciones con las variables fisicoquímicas (Figura 5). A nivel temporal se evidenció algún grado de relación con la profundidad, los coliformes totales, el caudal y la temperatura del agua $(\mathrm{p}<0,05)$.

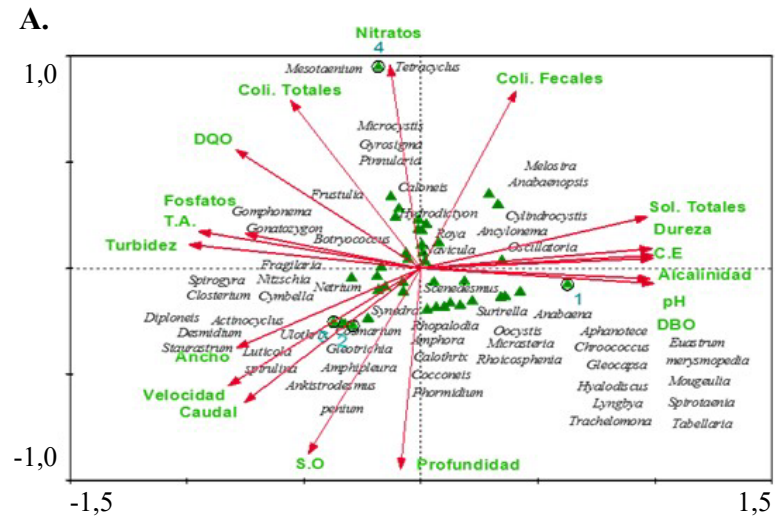

B.

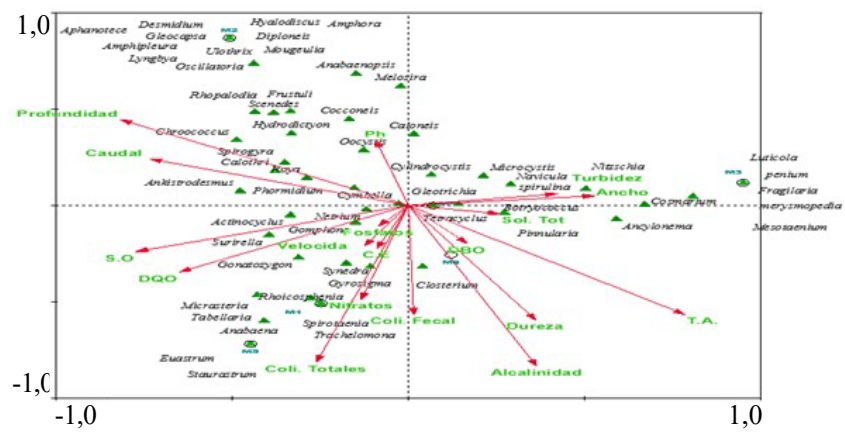

Figura 5. Análisis de correlación canónica entre las variables biológicas y las variables fisicoquímicas. (A) Estaciones; (B) Meses

\section{Discusión}

A nivel estructural, en todos los muestreos la división Bacillariophyta fue la más abundante y la Euglenophyta, la de menor abundancia. Estos resultados concuerdan con los obtenidos por Montoya \& Ramírez (2007), Bustamante, et al. (2008), Silva, et al. (2008), Pedraza \& Donato (2011) y Gordillo \& Reinoso (2013) en cuanto a que estas divisiones 
tuvieron una representatividad similar. Es muy probable que los organismos pertenecientes al filo Euglenophyta se encuentren de forma ocasional, ya que son característicos de ambientes con alto contenido de materia orgánica de origen natural, flujos y ambientes lénticos (Vilches, Casco, Giorgi, 2016), condiciones que se atribuyen principalmente a la estación E1, en donde la poca velocidad de la corriente y el material alóctono aportado por la vegetación ribereña crean las condiciones propicias para su establecimiento. La gran abundancia de la división Bacillariophyta podría estar relacionada con la segregación de estructuras mucilaginosas (Cymbella, Amphora, Cocconeis, Gomphonema, entre otros) que les permiten adherirse a diferentes sustratos y, así, mantenerse en las corrientes propias de un sistema lótico (Pinilla, 1998). Las diatomeas presentan diferentes tipos de movimientos según su morfología: las pennadas con rafe presentan movimientos espasmódicos, en tanto que la mayoría de las diatomeas céntricas y pennadas sin rafe rotan sobre un mismo punto, características que pueden generar procesos de deriva en la comunidad según cuán resistente sea su tipo de movimiento y adhesión (Allan, 1995). Además, tienen material silíceo en su pared celular (frústula), característica que les otorga mayor resistencia a cambios fisicoquímicos del medio (Bellinger \& Sigee, 2011).

Los géneros de diatomeas Synedra y Navicula presentaron altas abundancias y densidades relativas en todos los muestreos y en la mayoría de las estaciones; asimismo, el género Cosmarium fue representativo de la división Clorophyta y el Oscillatoria, de las cianobacterias, resultados que fueron similares a los obtenidos por Gualtero \& Trilleras (2001) en la represa de Prado. Estos autores establecieron que las mayores densidades poblaciones en la comunidad de algas estaba representada por las diatomeas, las clorofíceas y las cianofíceas, y que sus altas densidades podrían estar relacionadas con la naturaleza orgánica de diatomeas como Synedra y Navicula, las cuales presentan una frústula resistente (Bellinger \& Sige, 2011). Además, son géneros generalistas y cosmopolitas (Novelo, 2012), al igual que Cosmarium y Oscillatoria, caracterizados por ser cosmopolitas y por hacer parte natural de las comunidades de rocas en los ríos (Bellinger \& Sige, 2011).

El orden de riqueza (q:0) refleja que la riqueza fitoperifítica disminuye a medida que se avanza aguas abajo en el río; la medida de diversidad (q:1) evidencia el mismo comportamiento, siendo la estación E1 la que presentó la mayor diversidad, seguida de la estación E2, en tanto que hubo valores similares en las estaciones E3 y E4. Estos resultados evidenciarían cierto grado de intervención antrópica a medida que la altura disminuye, debido a que en sistemas con altas descargas y alteración del flujo, la diversidad y la riqueza tienden a disminuir (Clausen \& Biggs, 1996).

Las estaciones E2 y E3 registraron una gran relación entre sí, con un $70 \%$ de similitud, lo cual estaría asociado con la cercanía de cada punto y el grado similar de intervención antrópica, ya que estas dos estaciones son de uso recreativo y doméstico. Las estaciones E1 (nacimiento) y E4 (desembocadura) presentaron la mayor disimilitud entre ellas, sin embargo, la estación E4 (desembocadura) se diferenció de las demás, quizás porque esta zona presenta una gran influencia ganadera y vehicular a lo largo de su cauce, factores que modifican la química natural del agua y generan cambios en las dinámicas naturales de las comunidades perifíticas (Rivera, et al., 2010; Smucker \& Vis, 2011; Castro \& Pinilla, 2014).

Cabe resaltar que en este estudio, el análisis de escalamiento multidimensional no métrico (NMDS) demostró ser poco confiable en cuanto a su interpretación, probablemente por la diferencia en la naturaleza de las muestras tomadas, ya que los datos fisicoquímicos reflejaron una calidad de agua puntual, mientras que los datos biológicos fueron producto de cambios anteriores en la dinámica del cuerpo de agua, lo generó sesgo en los datos. En general, no se evidenció ningún tipo de asociación entre las comunidades biológicas y las variables ambientales.

\section{Conclusiones}

Se estableció que, a nivel temporal y espacial, hubo influencia del régimen hidrológico sobre las variables de porcentaje de saturación de oxígeno, $\mathrm{pH}$, temperatura del agua, profundidad, coliformes totales y caudal en la cuenca del río Anchique, variables que pueden incidir de forma directa o indirecta en el desarrollo de las comunidades fitoperifíticas.

A nivel temporal, la densidad y el número de géneros encontrados se vieron influidos, en parte, por la dinámica hidrológica del cuerpo del agua. Se registró la mayor densidad de perifiton en épocas de aguas bajas, siendo la temporada climática un dinamizador de la biota fitoperifítica del río.

Es importante hacer futuras investigaciones para evaluar el papel del perifiton en la dinámica trófica del ecosistema, así como para analizar su respuesta frente a eventos de perturbación natural a partir de sus rasgos funcionales, lo cual ofrecería una base para llevar a cabo biomonitoreos de estrés hídrico con las consecuentes implicaciones para la conservación y los planes y programas de manejo.

\section{Agradecimientos}

A la Universidad del Tolima, al Grupo de Investigación en Zoología y a todo el equipo técnico del macroproyecto del río Anchique, por su apoyo incondicional en campo.

\section{Contribución de los autores}

Kelly Huertas-Farías contribuyó en las salidas de campo, la determinación de las muestras y el análisis de datos; Gladys Reinoso corrigió el documento, y asesoró de forma continua en el proceso de determinación y tratamiento de las muestras; Yessica Tatiana-Parra colaboró con el análisis estadístico y con revisiones continuas de los procesos de cuantificación.

\section{Conflicto de intereses}

Ninguno. 


\section{Referencias}

Allan, D. (1995). Stream Ecology Structure and Function of Running Waters (primera edicion). Netherland: Kluwer Academic Publishers. p. 388.

Allan, D., Castillo, M. (2007). Stream Ecology Structure and Function of Running Waters (segunda edicion.). Netherland: Springer. p. 436.

American Public Health Association, American Water Works Association, Water Environment Federation. (2012). Standard Methods for the Examination of Water and Waste Water. American Public Health Association, 22 edición. Washington, United States: Academic Press. p. 1496.

Arcos, M. \& Gómez, A. C. (2006). Microalgas perifíticas como indicadoras del estado de las aguas de un humedal urbano: Jaboque, Bogotá D.C, Colombia. NOVA. 4: 60-79.

Bellinger, E. G. \& Sigee, D. C. (2011). Freshwater algae: identification and use as bioindicators. Oxford, UK: WileyBlackwell. p. 290.

Bicudo, C. \& Menezes, M. (2006). Géneros de algas de aguas continentales do Brasil (chave para identificaçao e descrições). Segunda edición. Brazil: Editora Rima. p. 489.

Biggs, B. J. \& Smith, R. A. (2002). Taxonomic Richness of Stream Benthic Algae: Effects of Flood Disturbance and Nutrients. Society of Limnology and Oceanography. 47 (4): 1175-1186.

Braak, C. J., Smilauer, P. (2004). Canoco Reference Manual and CanoDraw for Windows User's guide: Software for Canonical Community Ordination (version 4.5). Ithaca, New York: Microcomputer Power.

Brower, J. E. \& Zar, J. H. (1984). Field and laboratory methods for general ecology. Dubuque, Iowa: Wm. C. Brown Co. p. 226.

Bustamante, C. A., Dávila, C. A., Torres, S. L., Ortiz, J. F. (2008). Composición y abundancia de la comunidad de fitoperifiton en el Río Quindío. Rev.Invest.Univ.Quindío. 20: $15-21$.

Castro, D., Pinilla, G. (2014). Periphytic diatom index for assessing the ecological quality of the Colombian Andean urban wetlands of Bogotá. Limnetica. 33 (2): 297-312.

Clarke, K. \& Gorley, R. (2006). PRIMER v6: User Manual/ Tutorial. PRIMER-E, Plymouth, $192 \mathrm{p}$.

Clausen, B. \& Biggs, R. (1996). Relationships between benthic biota and hydrological indices in New Zealand streams. Freshwater biology. 38 (2): 327-342.

Di Rienzo, M., Balzarini, A., Cazanoves, L., González, M., Tablada, W., Guzmán, Robledo, W. (2013). InfoStat. Software estadístico. Manual de usuario. Universidad Nacional de Córdoba, Argentina: Grupo InfoStat. FCA.

Díaz-Quirós, C. \& Rivera-Rondón, C. A. (2004). Diatomeas de pequeños ríos andinos y su utilización como indicadoras de condiciones ambientales. Caldasia. 26: 381- 394.

Gómez, N., Donato, J., Adoni, G., Guash, H., Mateo, P., Sabater, S. (2009). La biota de los ríos: los organismos autótrofos. En Elosegui, A., Sabater, S. (Editores), Conceptos y técnicas en ecología fluvial. . Bilbao: Fundación BBVA. p. 85-96.

Gordillo, J. \& Reinoso, F. (2013). Composición y estructura de la comunidad fitoperifítica de la cuenca del río AlvaradoColombia. Tesis de pregrado. Ibagué. Colombia: Universidad del Tolima. p. 126.
Goring, D. G. \& Biggs, B. J. F. (1996). The effect of velocity and turbulence on the growth of periphyton in a cobblebed stream: The 5-stone experiment. In 2nd IAHR International Symposium on Habitat Hydraulics: A239-A250.

Gualtero, D. M. \& Trilleras, J. M. (2001). Estudio de la comunidad perifítica del embalse de Prado. Ibagué. Colombia: Universidad del Tolima. p. 193.

Guiry, M.D. \& Guiry, G.M. (2019). AlgaeBase. World-wide electronic publication, National University of Ireland, Galway. Fecha de consulta: junio a diciembre de 2016. Disponible en: http://www.algaebase.org

Harding, W.R., Archibald, C.G. Taylor, J.C. (2005). The relevance of diatoms for water quality assessment in South Africa: A position paper. Water SA. 31 (1): 41-46.

Hauer, R. \& Lamberti, A. (2007). Methods in Stream Ecology. USA: Academic Press. p. 506.

Hill, M.O. (1973). Diversity and Evenness: A Unifying Notation and Its Consequences. Ecology. 54: 427-432.

Krammer, K. \& Lange-Bertalot, H. (1997). Bacillariophyceae 2. Bacillariaceae, Ephithemianceae, Surirellaceae. Sussuaseflora von Mitteleuropa. 2: 1-611.

Kociolek, J.P., Balasubramanian, K., Blanco, S., Coste, M., Ector, L., Liu, Y., Kulikovskiy, M., Lundholm, N., Ludwig, T., Potapova, M., Rimet, F. (2016). DiatomBase. Fecha de consulta: junio a diciembre de 2016. Disponible en: http://www.diatombase.org

Lobo, E. A., Heinrich, C. G., Schuch, M., Wetzel, C. E., Ector, L. (2016). Diatoms as bioindicators in rivers. River Algae. 1: 245-271.

Lobo, E. A., Heinrich, C. G., Schuch, M., Heinrich, C. (2015). Development of the Trophic Water Quality Index (TWQI) for subtropical temperate Brazilian lotic systems. Environ Monit Asses. 187: 1-13.

Lopretto, E.\& Tell, G. (1995). Ecosistemas de aguas continentales. Argentina: Ediciones Sur, 1401 p.

Martínez, L. \& Donato, J. C. (2003). Efectos del caudal sobre la colonización de algas en un río de alta montaña tropical (Boyacá, Colombia). Caldasia. 25 (1): 337-354.

Masseret, E., Amblard, C., Bourdier, G. (1998). Changes in the structure and metabolic activities of periphytic communities in a stream receiving treated sewage from a waste stabilization pond. Water Research. 32 (8): 2299-2314.

Montoya, M. \& Ramírez, J. J. (2007). Variación estructural de la comunidad perifítica colonizadora de sustratos artificiales en la zona de ritral del río Medellín, Colombia. Revista de biología tropical. 55: 585-593.

Needham, J.G. \& Needham, P. R. (1978). Guía para el estudio de seres vivos de agua dulce. Traducción adaptada para España y América. Barcelona, España: De reverté. p. 131.

Novelo, E. (2012). Cyanoprokaryota. Fascículo 90 Serie: Flora del Valle de Tehuacán-Cuicatlán. Editor. R. Medina. Instituto de Biología, Universidad Nacional Autónoma de México. p. 96.

Patrick, R. (1988). Importance of diversity in the functioning and structure of riverine communities. Society of Limnology y Oceanography. 33 (6): 1304-1307.

Pedraza, E., Donato, J. (2011). Diversidad y distribución de diatomeas en un arroyo de montaña de los Andes colombianos. Caldasia. 33: 177-191.

Pinilla, G. (1998). Indicadores biológicos en ecosistemas acuáticos continentales de Colombia. Santa fe de Bogotá, Colombia: Fundación Universidad de Bogotá Jorge Tadeo Lozano, Centro de Investigaciones. p. 67. 
Pizarro, H. \& Alemanni, M. E. (2005). Variables físico-químicas del agua y su influencia en la biomasa del perifiton en un tramo inferior del río Luján. Asociación Argentina de Ecología. 15: 73-88.

Ramírez, J. (2000). Fitoplancton de agua dulce: bases ecológicas, taxonómicas y sanitarias. Colombia: Editorial Universidad de Antioquia. p. 207.

Ramírez, G. \& Viña, G. (1998). Limnología colombiana. Cap. 4, Sistemas Lóticos. Caracterización fisicoquímica II. Bogotá, Colombia: Exploration Company. p. 293.

Rivera, C. \& Donato, J. (2008). Influencia de las variaciones hidrológicas y químicas sobre la diversidad de diatomeas bénticas. En: Donato, J. (Editores) Ecología de un río de montaña de los Andes colombianos (río Tota, Boyacá). Bogotá D.C, Colombia: Universidad Nacional de Colombia. p. 83-102.

Rivera, C.A., Zapata, A.M., Pérez, D., Morales, Y., Ovalle, H., Álvarez, J.P. (2010). Caracterización limnológica de humedales de la planicie de inundación del río Orinoco. Orinoquía, Colombia. Acta biológica colombiana. 15 (1): 145-166.

Roldán, G. (1992). Fundamentos de limnología neotropical. Colombia. Universidad de Medellín, Colombia: Editorial Universidad de Antioquia. p. 529.

Roldán, G., Ramírez, J. (2008). Fundamentos de Limnología neotropical. (2.a ed.) Medellín, Colombia: Editorial Universidad de Antioquia. p. 440.

Silva, A. M., Sili, C., Torzillo. G. (2008). Cyanoprocaryota y microalgas (Chlorophyceae y Bacillariophyceae) bentónicas dominantes en ríos de Costa Rica. Revista Biología Tropical. 56: 221-235.
Smith, R. \& Smith, T. (2001). Ecología. 4a edición. Madrid, España: Addison Wesley. p. 642.

Smucker, N. \& Vis, M. (2011). Acid mine drainage affects the development and function of epilithic biofilms in streams. Journal of North American Benthology Society. 30 (3): 728-738.

Streble, H., Krauter, D. (1987). Atlas de los microorganismos de agua dulce. La vida en una gota de agua. Barcelona, España. Ediciones Omega, S.A. p. 372.

Tümpling, W. \& Friedrich, G. (1999). Methoden der Biologischen Wasseruntersuchung 2. Biologische Gewässeruntersuchung. 2: 310-331.

Vilches, C., Casco, M., Giorgi, A. (2016). El perifiton como indicador decontaminación difusa: el caso de la cuenca superior del río Reconquista (Buenos Aires, Argentina). Boletín de la Sociedad Argentina de Botánica. 51 (4): 579-595.

Weber, C. (1968). The preservation of phytoplankton grab samples. Transactions of the American Microscopical Society. 87: 70-81.

Wehr, J.D. \& Sheath, R.G. (2003). Freshwater Algae of North America: Ecology and Classification. USA, Academic Press. p. 1066.

Wu, J.T. (1999). A generic index of diatom assemblages as bioindicator of pollution in the Keelung River of Taiwan. Hydrobiologia. 397: 79-87

Zapata, A. M. \& Donato, J. C. (2005). Cambios diarios de las algas perifíticas y su relación con la velocidad de corriente en un río tropical de montaña (río Tota-Colombia). Limnetica. 24: 327-338. 